\title{
Acquired Ocular Toxoplasmosis in Diabetic Pregnancy - a Hidden Monster
}

Authors: Dr Ashish Dengra ${ }^{1}$, Dr Jigyasa Dengra ${ }^{1}$, Dr Rahul Choubey ${ }^{2}$, Dr Rajesh Jain ${ }^{3}$

Institutions: 1. Mahi Diabetes, Thyroid Care and Research Centre, Jabalpur, MP, India. 2. Rashmi Eye Centre, Jabalpur, MP, India. 3. Jain Hospital, Kanpur, UP, India
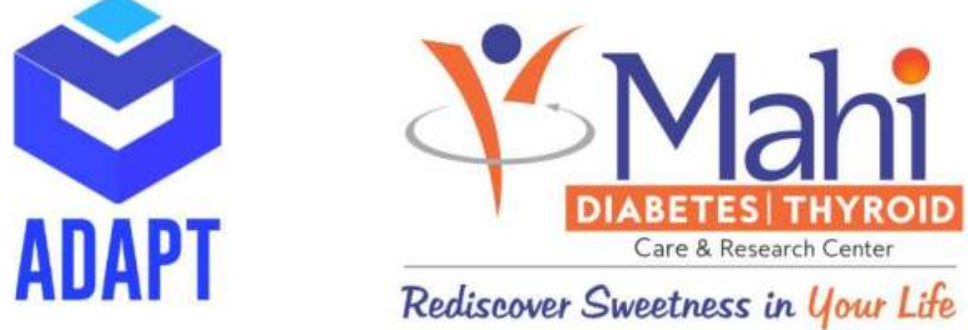

Rediscover Sweetness in Your Life

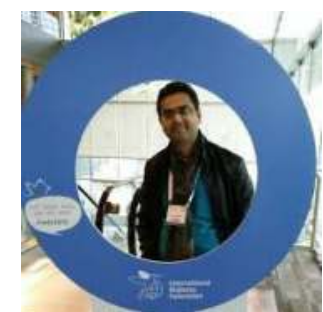

\section{Background}

- In India ocular toxoplasmosis is expected to occur through the congenital route, with an index of underestimation for the incidence through the acquired route

- Overall, about $40 \%$ of primary maternal infections lead to congenital infection of children, with a transplacental transmission rate of $\mathrm{T}$ gondii reported to be $3 \%$ in the first trimester, $22 \%$ in the second, and $63 \%$ in the third.

- Ocular toxoplasmosis in pregnancy has been reported in the literature

- Reactivated latent ocular toxoplasmosis during pregnancy may result in decreased vision and floaters

- Toxoplasmic retinochoroiditis is the most common cause of posterior uveitis in immunocompetent patients

- However, acquitted toxoplasmosis is not reported in pregnant women with diabetes

\section{Aims}

We describe the management of a case of recurrent ocular toxoplasmosis that presented in the first trimester of pregnancy in a diabetic patient

\section{Presentation}

- 27 year old apparently healthy, at 9 weeks' gestation, presented with a 1 week history of blurred vision in the left eye. + ve $\mathrm{h} / \mathrm{o}$ past ocular problems

- First complicated pregnancy was two years ago which resulted in still born child

- There was a history of cattle at home

\section{Clinical Examination}

- Best corrected visual acuities were 6/6 and 6/36 in Right (RE) and Left Eye (LE)

- Anterior segments were normal and the intraocular pressures were $16 \mathrm{mmHg}$ RE, $14 \mathrm{mmHg}$ LE

- Fundoscopy findings- diffuse, elevated white lesion in the left retina half a disc diameter in size located one disc diameter superior to the fovea (Fig 1)

- An area of associated perivascular sheathing and minimal vitreous activity was noted

- An active left retinochoroiditis with associated retinal vasculitis was diagnosed. The right fundus was normal

\section{Serological testing}

- Revealed antibodies to Toxoplasma gondii at a titre of $1 / 4096$ (2000 IU/ml) using the dye test

- Toxoplasma IgM enzyme linked immunosorbent assay (ELISA) on the patient's peripheral blood was positive

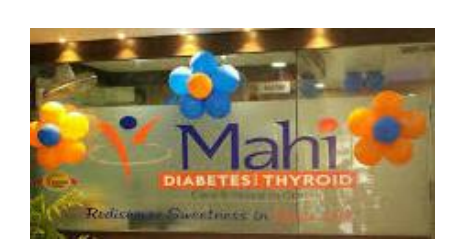

Copyright $\odot 2019$

Dr Ashish Dengra

E: drashishdengra@hotmail.com
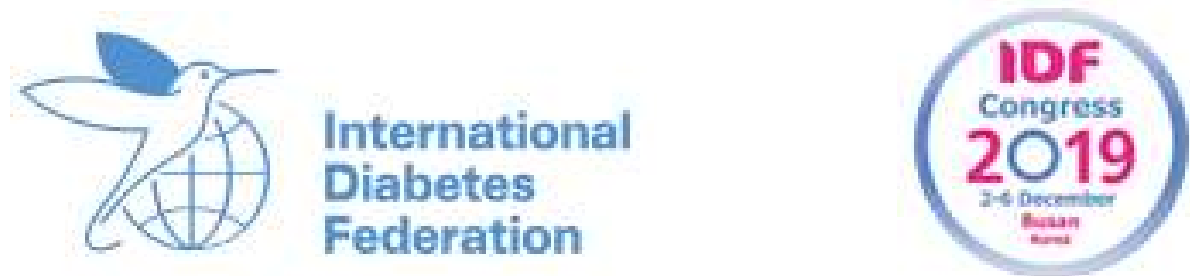

P-0762

\section{Follow Up and Management Course}

- At three weeks after presentation (12 weeks gestation) the visual acuities remain unchanged. However, there was now occlusion of a small retinal arteriole crossing the lesion

- After consultation with the obstetrician and risk-benefit assessment the patient decided to proceed with the pregnancy

- Therapy initiated - oral spiramycin $1 \mathrm{~g}$ three times daily

- Eight weeks after presentation (17 weeks gestation) the vision remained the same and the focus of retinochoroiditis was beginning to scar

- Spiramycin continued until the end of pregnancy

- Sequential follow up fetal ultrasounds were normal

- At term the patient gave birth to a healthy baby girl

\section{Post Partum}

- Polymerase chain reaction (PCR) and culture for T gondii were negative on placental tissue.

- As toxoplasma IgM ELISA, IgM, and IgA ISAGA on cord blood were negative, the positive dye test was probably detecting passively transferred maternal $\mathrm{IgG}$

- The mother's vision remained normal, and only a small, pigmented scar was present (Fig 2).

Fig 1

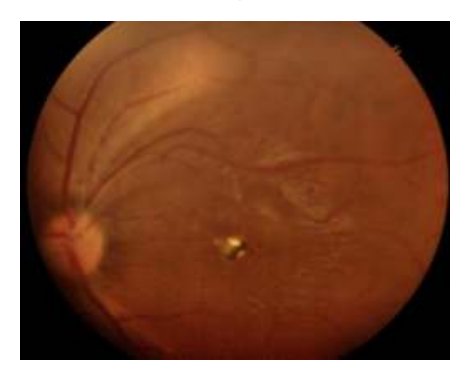

\section{Discussion}

- In our case the features supporting an acquired aetiology, the fresh perspective, included lack of retinal scars from previous infection, and the positive IgM serology and IgG avidity results

- Spiramycin was effective in reducing the risk of transmission to the fetus which may have contributed to the resultant good outcomes, both for both mother and fetus

- Determining an aetiology of acquired T gondii infection was important in this patient as it allowed the most appropriate management plan to be initiated resulting in an excellent outcome

- Recurrent ocular toxoplasmosis occurring in pregnancy in a diabetic patient is threatening both to patient and child

- Prompt vigilance with correlation with past history and fundus examination is important

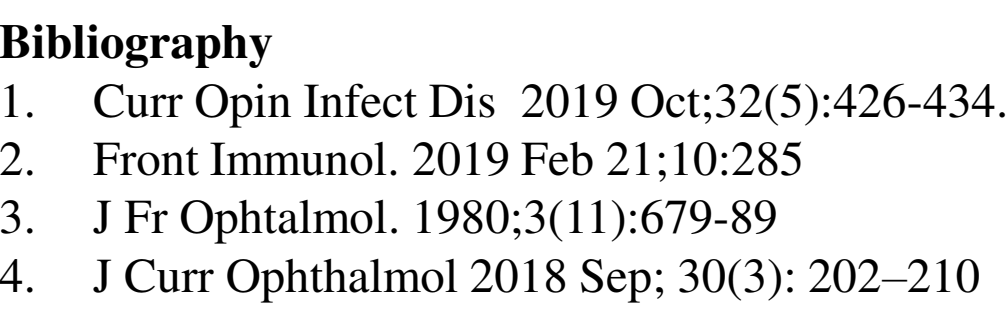

